\title{
Development of System to Detect Dying Alone for Indigent
}

\author{
${\text { Moeko Konomi }{ }^{\text {a,* }} \text {, Fumiaki Ehara }}^{\text {b }}$, Kazuo Haruyama ${ }^{\mathrm{b}}$, Yuhki Kitazono ${ }^{\mathrm{a}}$ \\ ${ }^{a}$ Kitakyushu National College of Technology,5-20-1 Shii, Kokuraminami-ku, Kitakyushu-city, \\ Fukuoka 802-0985, Japan \\ ${ }^{\mathrm{b}}$ Ube National College of Technology, 2-14-1 Tokiwadai, Ube-city, Yamaguchi 755-8555, Japan \\ *Corresponding Author: d31022mk@apps.kct.ac.jp
}

\begin{abstract}
In Japan, the number of aged single persons who are found dead late has been increasing year after year. To prevent that, they and their family can use the system which sends a notice to the family's smartphone with the internet at present. However, if the people mentioned above is single and indigent, they can't use that system because low-income or solitude.

So, we developed the system to detect dying alone for indigent as well as related apparatus. Our system can send a notice to the caretaker or administrative organ without the internet. This system has a parent device and some children devices. Caretakers have a parent device, then users each have a child device. It is configured by some microcomputer, passive infrared pyroelectric sensor, as many "X-Bee module" and so little energy. The system is as follows: First, users attach a child device to the lavatory. When people go to there, this system gets time. Next, the child device sends information to the parent device without the internet connection by X-Bee module. This merit is simple Installation and low price. From this, even though old people can't use system with the internet from indigent, caretakers use our system for such people and prevent problems easily.
\end{abstract}

Keywords: dying alone, microcomputer, X-Bee module.

\section{Introduction}

In Japan, the recent elderly people who live alone have been increasing year by year. Because of aging population and lower birthrate, nuclear families have been advancing ${ }^{(1)}$. This study we take up, the case which the people found dead late after dying alone in their home. Such case is regarded as a question from it not only damages deceased person's honor, but also hurt discover and the caretaker mentally. To prevent that, there are various systems and community involvements ${ }^{(2,3)}$.

Currently, there are the following systems for watch the aged. They are bought and used by their family. The systems send a notice to the family's phone. Basically they are using the internetwork or the telephone line, and moreover, those take many costs. So it is difficult to say that those systems can install easily for everyone ${ }^{(4)}$.

On the other hand, there are also community involvements to prevent the matter. Their activity is to visit and watching over elderly by neighborhood at stated periods. This way is a valid in the country which inhabitants on neighborly terms well of there. But it is difficult in populous urban area. Therefore it is hard to carry it out nationwide.

Accordingly, our study aim to develop the watching system for caretakers for a public housing in which aged indigents live. The system we propose can use without internet due to radio communication module. Thereby, it can be to simply install the device. And the caretakers use the system without feeling it in a burden.

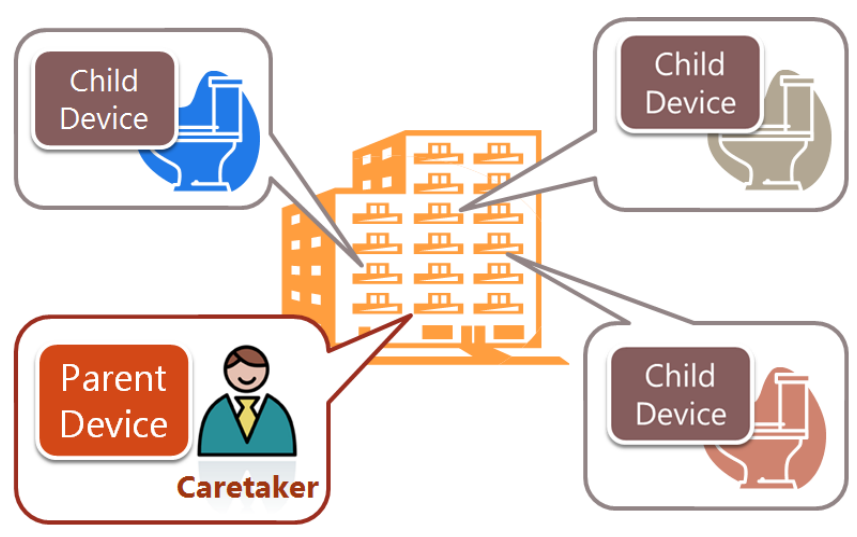

Fig. 1. System Configuration. 


\section{System Configuration}

As in the fig.1, this system has a parent device and some children devices. Caretakers have a parent device, then elders each have a child device. Those details are given as under.

\subsection{Pyroelectroc Sensor}

Our device used the pyroelectric sensor produced by Parallax Inc. This sensor is moving object detection module and detects motion of animals that have a body temperature. According to specifications, detection distance of the sensor is about $7 \mathrm{~m}$, detection angle is 110 degree and operating voltage is DC $5 \mathrm{~V}$. This sensor is used for the purpose of detection that the human went to the lavatory. In this condition range, it can be used in the place that user does not see in usual like ceiling of lavatory ${ }^{(5)}$. For these reason, we selected the sensor for use in this device.

\subsection{Radio Communication}

We use XBeeZB module for radio communication. Owing to use this module, the system can run in the housing without internet line. This module has feature that is low cost, and the module can consist low-power mesh network. According to specifications, the communication distance is $40 \mathrm{~m}$ inside the room, or $120 \mathrm{~m}$ in the air. In spite of that, communicating with mesh network using multiple children devices like fig.2, it can communicate with each other over more distant than specification distance. Owing to this, the device can get enough distance to use in place like public housing of low income elder.

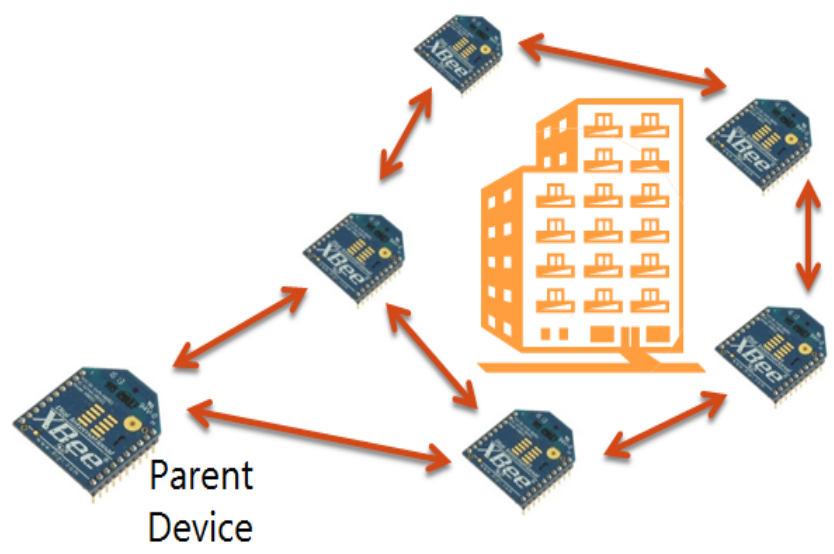

Fig. 2. Mesh Network.

\subsection{Child Device Program}

Devices of system use PSoC produced by Cypress Semiconductor Inc. We used PSoC Designer software as development environment. Children devices consist of a microcomputer, pyroelectric sensor and X-Bee module. The child device always measures time. When pyroelectric sensor detects a person the timer is reset and measures the passage of the time until detects a person again. It always repeats that and when it receive a signal from parent device, returns the number of the device and the elapsed time at the moment.

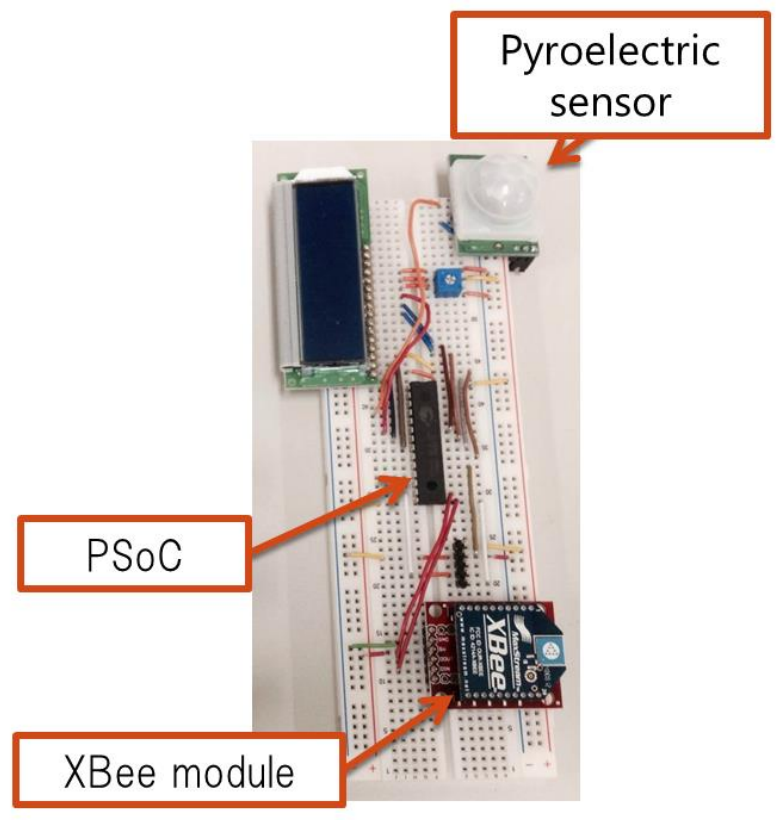

(a) Child Device

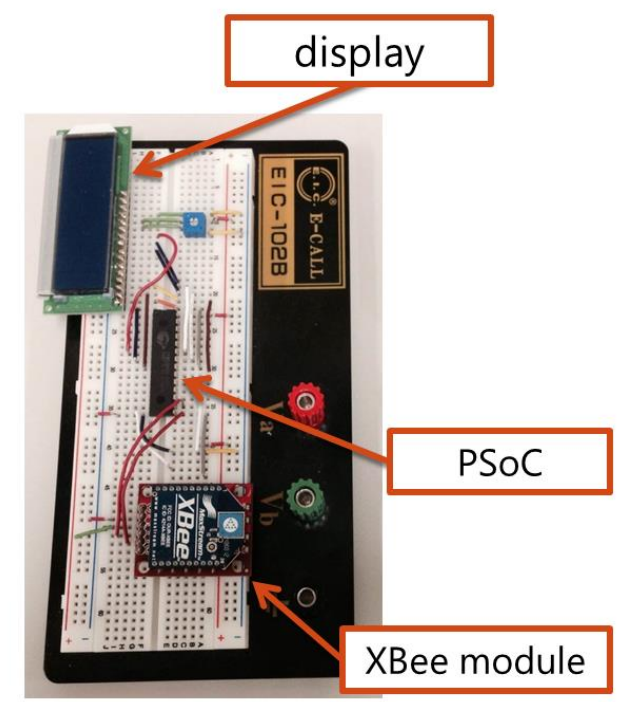

(b) Parent Device

Fig. 3. Devices. 


\subsection{Parent Device Program}

Parent device consists of a microcomputer, display and $\mathrm{X}$-Bee module. It sends a signal to each children devices every 12 hours. It displays the elapsed time that the parent device obtained as a response from the child device. When caretaker looks the time, he judges the existence/ absence of abnormality.

\section{Experiment}

\subsection{Pyroelectric Sensor}

We examined whether the pyroelectric sensor used in this device is adequate to embed in the device in practice. We examined the pyroelectric sensor, using display and program of microcomputer.

To examine the pyroelectric sensor, we made a program that shows "ON" on monitor when the pyroelectric sensor detected some motion of a person, and shows "OFF" when it doesn't. The purpose of this examination is to determine whether sensor performs well or not, and how long the range of sense is.

This experiment yields that the pyroelectric sensor proved to have $5 \mathrm{~m}$ range of detection to sense a motion of person. In addition to this, though a person is in the range of $5 \mathrm{~m}$, the pyroelectric sensor doesn't detect anything when the person makes no motion (Fig.4). Therefore, the sensor can be set to the place where people does not look in usual such as ceiling of lavatory due to the range of $5 \mathrm{~m}$ the sensor has. Besides, it is proved that the sensor can detect an abnormality when the person become unable to move because the pyroelectric sensor detects nothing when the person doesn't move even though is in range of sense.

\subsection{System Validation}

We examined system validation. We examined system, using a parent device and a child device.

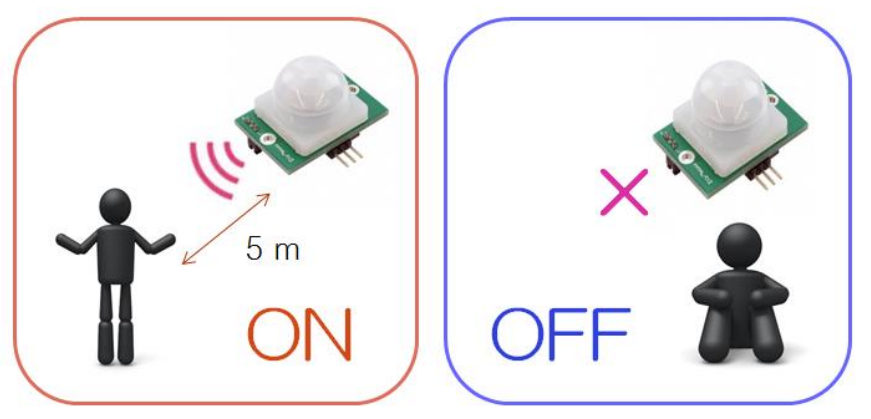

Fig. 4. Pyroelectric Sensor.
To examine the system, we made a program that send signal to a child device from a parent device, after that, a child device send a message to a parent device as a response. We installed the child device at celling of the room 1 and put the parent device in the room 2 next to room1. The parent device sends a signal every two minutes. Then the child device sends the elapsed time. When the parson enters the room1, the child device resets the time measurement and starts it again.

As a result, when the parson entered the room1, the child device sent the elapsed time which was reset and restarted to parent device. In addition when the parson didn't enter the room1, the child device sent the elapsed time from the time when last the child device detected the parson. This shows pyroelectric sensor and programs ran normally. The experiment yields that the system can run inside the room.

\section{Discussion}

In this study, we made monitor system that run without internet line. Due to this system is low cost and simple, it will not be difficult that installed the housing that low income elders are living. However, mesh network needs to use many children devices, and therefore it has to get the understanding and cooperation of even more elders, and install even more devices. As the future issues is to make the system that can communicate regardless of an environmental scale. Similarly we should make displaying easy to understand. At present children devices are large in size owing to the display for experiments. We need to make them smaller to be really usable, and it will be necessary to improve it at a more practical device in future.

\section{Conclusions}

Japan will make progresses of medical technology and keep its peace. The number of elders will steadily increase. Furthermore, the young in today will become elder for time to come, and due to that comparative most of them don't marry, aged single person will more and more increase. Aged person who is not engaged in an occupation is supported to live by the government and most of them will live in public housing. We studied to prevent the management of such place from making him feel uncomfortable, and to make useful device to discover emergency like dying alone.

Even though the internet has spread, some people are 
left for some reason such as low income or an advanced age. The device we developed may be not as speedy in discovering as the system provided by security companies or patrols by neighborhood. Even so, there would be enough usage value because it can prevent low income aged person or caretaker of them from being in a miserable situation.

We expect to reduce even one person who suffered from dying alone by using this device.

\section{References}

(1) Cabinet Office, Government of Japan : “2009 Aging Situation and Enforcement Situation of Measures to Aged Society", 2010 White Paper on Aged Society, pp.52-73, 2010

(2) Chizuru Saito : "A Study of Care System for old people", Journal of Kansai University of Welfare Sciences, Vol.13, pp.175-188, 2010

(3) Noboru Ichida, Nobuayoshi Fujimoto, Eiji Satho, Nobuo Mitsuhashi, Hiroyuki Honjo, Takashi Shimoji, and Tomoe Miura : “6059 On Welfare Officer's Activities for the Aged : A Study on Local Supporting System for the Self-reliance Aged Accordance with Their Living Conditions", Summaries of technical papers of Annual Meeting Architectural Institute of Japan. E-2, Architectural planning and design II, Dwelling houses and housing sites, rural planning, education, Vol.2011, pp.515-516, 2011

(4) Akihiro Sakai, Masataka Kitama, Kazutuki Kimura, and Junji Arisawa : "Support System for isolated elderly people using sensors and the network equipment", IEICE Technical Report, Vol.110, No.354, pp.29-33, 2010

(5) Yoshimitsu Shinagawa, and Hayato Hashimoto : "Development of Home Health Monitoring System for the Elderly after much Consideration of Humanity", Kawasaki Journal of Medical Welfare, Vol.11, No.1, pp.199-204, 2001 